\title{
Machtkonstellationen im Schwarzmeerraum - Implikationen eines EU-Beitritts der Türkei und anderer Anrainer
}

\author{
Sammi Sandawi*
}

Seit der Aufnahme von Beitrittsverhandlungen mit der Türkei am 3. Oktober 2005 ist die Europäische Union (EU) mehr denn je gezwungen, sich auch mit ihrer zukünftigen Rolle im Schwarzmeerraum auseinanderzusetzen. War das Gebiet um das Schwarze Meer bisher aus Brüsseler Perspektive sowohl geographisch als auch geopolitisch weitgehend peripheres europäisches Terrain, lässt sich mittlerweile auf Seiten der EU ein wachsendes Interesse am euro-asiatischen Grenzraum verzeichnen. Neben den sechs Anrainern Bulgarien, Rumänien, Ukraine, Russland, Georgien und Türkei umfasst das Gebiet ein bis zu 20 Millionen Quadratkilometer großes Territorium, welches 14 Staaten, 330 Millionen Einwohner sowie ein Außenhandelsvolumen von 300 Milliarden US-Dollar in sich vereint. Zugleich repräsentiert der ,Erweiterte Schwarzmeerraum' das weltweit zweitgrößte Reservoir an fossilen Energieträgern (namentlich Erdöl und Erdgas), deren Transit in die Hauptabnahmeländer Westeuropas und Nordamerikas zu einem Großteil über den unmittelbaren Schwarzmeerraum abgewickelt wird. ${ }^{1}$

\section{Die EU-Erweiterung als Pforte in die Region}

Darüber hinaus ist eben jenes Kerngebiet traditionell Austragungsort zahlreicher regionaler Konflikte, dem mit der bevorstehenden sukzessiven EU-Erweiterung eine neuerliche machttektonische Verschiebung droht. So birgt die - sich spätestens mit dem türkischen Beitritt vollziehende - Erhebung der EU in den Rang einer „Black Sea Power“2 ein erhebliches Störpotenzial für die Sicherheitsbalance, welche sich gegenwärtig in einem bilateralen Rapprochement der einst erbittert um Hegemonie ringenden Mächte Türkei und Russland widerspiegelt. Verbindendes Element beider Staaten ist dabei das gemeinsame Streben nach einer umfassenden Abschottung des Schwarzen Meeres gegenüber ,raumfremden Mächten“3. Diese Bemühung um eine - in erster Linie gegen die Vereinigten Staaten gerichtete Exklusionspolitik wird jedoch durch die stark transatlantisch ausgerichtete Politik Bulgariens und Rumäniens konterkariert, die sich im Rahmen bilateraler Stationierungsabkommen zu weit reichenden Zusagen gegenüber den USA bei deren Machtprojektion in den Schwarz-

\footnotetext{
Sammi Sandawi, Dipl. SozWiss., Institut für Europäische Politik, extern, Berlin.
}

1 Eine Übersicht zum weltweiten Energietransit findet sich in: Directorate-General Energy and Transport: Study on Energy Supply Security and Geopolitics, European Commission, Brussels, January 2004, S. 237-238, abrufbar unter: http://europa.eu.int/comm/energy_transport/doc/2004_lv_ciep_report_en.pdf.

2 Mustafa Aydin: Europe's next shore: the Black Sea region after EU enlargement, EU Institute for Security Studies, Paris, June 2004, S. 3.

3 Der bereits auf Carl Schmitt zurückgehende Begriff der ,,raumfremden Macht“ basiert auf der Annahme, dass die jeweils zwischen Großmächten liegenden Territorien einer nahezu naturgesetzlichen Spannung unterliegen, was im Schnittbereich konkurrierender Einflusssphären durchaus in aktiven Konflikten kumulieren kann. Erst das politisch-militärische Engagement einer dritten, im jeweiligen ,Großraum' nicht originär beheimateten (raumfremden) Interventionsmacht stellt jedoch die regionale Balance nachhaltig in Frage und führt so zu einer verschärften Konflikteskalation. Vgl. Carl Schmitt: Völkerrechtliche Großraumordnung mit Interventionsverbot für raumfremde Mächte. Ein Beitrag zum Reichsbegriff im Völkerrecht, Berlin 1939. Neuere raumbezogene Ansätze finden sich etwa in: Heinz Brill: Geopolitik heute: Deutschlands Chance?, Frankfurt am Main/Berlin 1994. Grundsätzlich: Herfried Münkler: Über den Krieg. Stationen der Kriegsgeschichte im Spiegel ihrer theoretischen Reflexion, Weilerswist 2003. 
meerraum bereit fanden. So öffnet sich für die EU mit der bis spätestens 2008 vollendeten Erweiterung um die beiden Balkanstaaten nicht nur eine erste Pforte in die Region, sondern möglicherweise auch ein neues Kapitel transatlantischer Auseinandersetzungen. Jenseits dieses Wettstreits um regionale Hegemonie, gibt vor allem die Situation der beiden ,post-revolutionären' Transformationsstaaten Georgien und Ukraine Anlass zur Sorge. So sehen sich diese nicht nur in einem latenten Konflikt mit dem ungleich größeren Nachbarn Russland involviert. Sie befinden sich zudem in einem Stadium ungesicherter bündnispolitischer Einbindung. Erschwert wird die Situation durch die vitale Abhängigkeit beider Staaten von russischen Energielieferungen, was im Januar 2006 keineswegs erstmals zu einer offenen Krise führte ${ }^{4}$. Als weitere Herausforderung in der Region stellt sich zudem die Tatsache dar, dass nahezu alle Volksgruppen im Schwarzmeerraum in Folge der Jahrhunderte währenden osmanischen Besatzung auf eine überaus konfliktbeladene Geschichte zurückblicken. Deren Ausläufer schlagen sich bis heute in den Perzeptionen der Anrainer nieder, belasten die bilateralen Verhältnisse zur Türkei, was wiederum für die Gemeinsame Außen- und Sicherheitspolitik (GASP) und die Europäische Nachbarschaftspolitik (ENP) der Union bedeutsam ist.

Dessen ungeachtet kam es spätestens seit Ende der neunziger Jahre auf Initiative der Türkei zu einer politischen und strategischen Annäherung zwischen den Schwarzmeeranrainern. Erste - wenngleich bisher in ihrem Wirkungskreis äußerst beschränkte - institutionelle Ausläufer dieser Entwicklung sind die ,Black Sea Economic Cooperation Organization' (BSEC), welche sich neben Handelsfragen auch mit dem Aspekt der regionalen ,Soft Security' auseinandersetzt sowie die ,Black Sea Naval Cooperation Task Group' (BLACKSEAFOR), die erstmals die maritimen Militärfähigkeiten der Anrainerstaaten zum Zwecke der regionalen Sicherheit zu bündeln sucht. Hintergrund der vorläufig zögerlichen Bereitschaft der Schwarzmeeranrainer zur regionalen Kooperation ist unter anderem auch die Befürchtung, dass sich durch eine zu effektive regionale Sicherheitsvorsorge gleichzeitig die eigenen Beitrittschancen zur Nordatlantischen Allianz sowie zur Europäischen Union schmälern könnten. Denn die Perpetuierung regionaler Instabilitäten in Europas ,schwierigste[r] Nachbarschaftsregion" ${ }^{5}$ katalysiert zugleich die Aufmerksamkeit und Hilfsbereitschaft beider Organisationen. Auf der anderen Seite versucht die EU vor allem im Rahmen ihrer ENP eine Stärkung regionaler Kooperationsstrukturen zu befördern und sieht im Schwarzmeerraum besonders für die BSEC ,an important part to play“6. Um dessen Effektivität zu steigern, wird seitens der EU bereits seit mehr als einem Jahr an der institutionellen Verknüpfung zwischen beiden Organisationen gearbeitet und soll unter anderem durch einen ständigen Beobachtersitz der EU bei der BSEC erfolgen. ${ }^{7}$

So sieht sich die EU im Schwarzmeerraum mit einem komplexen Beziehungs- und Interessengeflecht konfrontiert, ohne sich bisher in vollem Umfang über ihre eigenen Ambitionen und Interessen im Schwarzmeerraum bewusst geworden zu sein. Hinzu kommt, dass die Union bisher aufgrund der Heterogenität der Anrainerstaaten sowie der mit ihnen verbunde-

4 Exemplarisch im Falle Georgiens siehe: Klaus-Helge Donath: Trotzig gegen „Gasputin“, in: Neue Zürcher Zeitung, 29. Januar 2006. Für die Ukraine: Anatol Lieven: The West's Ukraine Illusion, in: International Herald Tribune, 6. Januar 2006.

5 Uwe Halbach: Der Kaukasus in neuem Licht. Die EU und Rußland in ihrer schwierigsten Nachbarschaftsregion, Stiftung Wissenschaft und Politik, Berlin, November 2005, S. 5.

6 European Commission, "European Neighbourhood Policy Strategy Paper", Communication from the Commission to the European Communities, COM(2004) 373 final, Brussels, 12. Mai 2004, S. 21.

7 Zu den unterschiedlichen bilateralen Kooperationsvorhaben siehe: Towards Strengthening EU-BSEC Relations - The Work Programme of the Hellenic Republic during it's Chairmanship of BSEC, in: Black Sea Trend Review Nr. 9/2005, S. 66-68. 
nen Interessen und Probleme ein reiches, aber nicht abgestimmtes Instrumentarienset zur Anwendung brachte: Eine Strategische Partnerschaft und Politik der vier Räume mit Russland, eine Europäische Nachbarschaftspolitik gegenüber der Ukraine und Georgien sowie Beitrittsverhandlungen mit Bulgarien, Rumänien und der Türkei. ${ }^{8}$ Zusammen mündet dies jedoch nicht in eine kohärente Strategie im Umgang mit der Gesamtregion. Dass die EU dessen ungeachtet in wachsendem Maße über strategische Interessen in der Region verfügt, weshalb die Union langfristig zu einem umfassenden Politikansatz gegenüber der Region finden sollte, wird im Anschluss an die folgende Betrachtung der geopolitischen Konfiguration des Schwarzmeerraums sowie der aktuellen Konflikte und Kooperationsanstrengungen erörtert.

\section{Die Konfiguration des Schwarzmeerraums: Historische und geopolitische Determi- nanten}

Die Schwarzmeerregion bildet aus Sicht ihrer Anrainer ein historisch überaus sensibles Terrain, welchem in der Vergangenheit unterschiedliche sicherheitsbezogene Funktionen zufielen. Abstrahiert man dabei von den sich aktuell darbietenden Beziehungsmustern, ist der Schwarzmeerraum im Sinne Halford Mackinders eine geopolitische „Pivot Area“.9 Sie diente mitteleuropäischen Mächten seit jeher als Durchgriffsraum auf die unterschiedlichen Ressourcen Zentralasiens. Sie ist gleichzeitig als Scheitelpunkt westlicher, östlicher und islamischer Kultur von mehreren Huntingtonschen ,Fault-Lines ' durchzogen. ${ }^{10}$ Dass diese Raumkonfiguration eine ,strukturelle Konfliktträchtigkeit“ aufweist, belegen mehrere im erweiterten Schwarzmeerraum anzutreffender, Frozen Conflicts ${ }^{` 11}$, von denen einige in der Vergangenheit - ebenso wie der tschetschenische Separationskonflikt - aufgrund vorhandener Stellvertreterstrukturen auch auf das türkisch-russische Verhältnis ausstrahlten.

\section{,Turkish Yoke' und Minderheitenfrage}

Noch heute weist der gesamte Küstenstreifen des während der Jahrhunderte langen osmanischen Herrschaft als ,Ottoman Lake' bezeichneten Gewässers zahlreiche Spuren der einstigen Besatzungszeit auf, die sowohl auf türkischer als auch auf ehemals besetzter Seite in erheblichem Maße die raumbezogenen Perzeptionen und Interessen der Gegenwart beeinflussen. Vor allem in Bulgarien, aber auch in Rumänien ist der Eindruck des nahezu fünfhundert Jahre andauernden ,Turkish Yoke' eine schmerzhafte, tief im kollektiven Bewusstsein eingebrannte Epoche, deren Verarbeitung keineswegs als abgeschlossen betrachtet werden kann. Auf der anderen Seite leben auf dem ehemals osmanischen Gebiet bis heute zahlreiche türkischstämmige Volksgruppen in der Diaspora, die in der sowjetisch dominierten Epoche bisweilen erhebliche Repressionen zu erdulden hatten und als deren Anwalt sich Ankara versteht. ${ }^{12}$ Allein in Bulgarien stellt die türkischstämmige Minderheit rund 9,4\% der Gesamtbevölkerung, deren politische Vertretung (,Movement for Rights and Freedoms') so-

8 Vgl. zur Problematik der Heterogenität von EU-Instumenten im Schwarzmeerraum: Marius Vahl: The EU and Black Sea Regional Cooperation: Some Challenges for BSEC, Centre for European Policy Studies, 15. April 2005, abrufbar unter: http://www.ceps.be/Article.php?article_id=420.

9 Vgl. Halford John Mackinder: The Geographical Pivot of History, in: Geopolitical Journal 23 (1904), S. 421 444.

10 Vgl. Samuel Huntington: Kampf der Kulturen: Die Neugestaltung der Weltpolitik im 21. Jahrhundert, München 1996, S. 455-460.

11 Hierzu zählen sowohl der Streit zwischen Moldau und Russland um Transnistrien, die russisch-georgische Auseinandersetzung um die Teilrepubliken Abchasien und Süd-Ossetien sowie der armenisch-aserbaidschanische Antagonismus um Nagorny-Karabach.

12 Eine ausführliche Würdigung findet sich u.a. in: Georg Brunner: Nationalitätenprobleme und Minderheitenkonflikte in Osteuropa - "Strategien für Europa", Gütersloh 1996. 
gar an der aktuellen sozial-liberalen Regierungskoalition beteiligt ist. Trotz dieses (für das beiderseitige Verhältnis positiv zu wertenden) Umstandes hat die letzte Parlamentswahl in Bulgarien allerdings verdeutlicht, dass eine wachsende Bevölkerungsgruppe um die rechtspopulistische, explizit anti-türkische Ataka-Partei (8\%) sich bewusst gegenüber der vorwiegend muslimischen Minderheit abzugrenzen sucht und dieser vor allem nach den Ereignissen des 11. September 2001 zunehmend feindselig gegenübersteht. ${ }^{13}$

Auch im Verhältnis zur Ukraine bildet die Frage der noch heute auf der Krim lebenden türkischstämmigen Minderheit einen latenten Streitpunkt mit der Türkei. Beklagt wird von türkischer Seite vor allem die zu geringe wirtschaftliche Unterstützung der überwiegend in ärmlichen Verhältnissen lebenden Krimtataren durch die Regierung in Kiew sowie deren nicht erfolgte rechtliche Gleichstellung. Hinzu kommt, dass die neue Regierung in Kiew seit der (auch von der türkischstämmigen Minderheit unterstützten), Orangen Revolution' zunehmend versucht ist, die Krimtataren gegen die ungeliebte russische Bevölkerungsmehrheit auf der ukrainischen Halbinsel auszuspielen.

Auch machtpolitisch spielte die Minderheitenfrage in der Vergangenheit eine wichtige Rolle, da diese der Türkei angesichts der russischen Schwäche nach dem Zusammenbruch der Sowjetunion ,die einmalige Chance [bot], eine Stellung als regionaler Hegemon [...] einzunehmen. " ${ }^{14}$ So verfolgte die Türkei vor allem unter ihrem Staatspräsident Turgut Özal den Aufbau einer von Ankara gelenkten pantürkischen Allianz, welche sowohl die im europäischen als auch im zentralasiatischen Raum lebenden Volksgruppen unter türkischer Führung zu vereinen suchte. Allerdings weckte die gegenüber den Schwarzmeeranrainern äußerst provozierende türkische Attitüde zu Beginn der neunziger Jahre auch auf Seiten einzelner Turkvölker Befürchtungen vor dem Heranwachsen eines neuen ,Großen Bruders', was das türkische Streben nach regionaler Vorherrschaft untergrub und nachfolgende Regierungen zwang, ausdrücklich Abstand von diesem Vorhaben zu nehmen.

\section{Energietransit und Versorgungssicherheit}

Die zweite, den Schwarzmeerraum maßgeblich determinierende Größe ist die der fossilen Energieressourcen. Zwar finden sich im Schwarzen Meer lediglich geringe Mengen Erdöl, doch ist die Region unter dem Gesichtspunkt des Transits kaspisch-zentralasiatischer Brennstoffe nicht nur aus der Perspektive der vornehmlich westeuropäischen Empfängerstaaten von zunehmend vitaler Bedeutung. ${ }^{15}$ Vielmehr ergibt sich aus Sicht der Anrainerstaaten sowohl die Chance kurzfristiger ausländischer Direktinvestitionen als auch langfristiger Erträge aus den Durchleitungsgebühren, was die strukturschwachen Schwarzmeerländer in eine natürliche Konkurrenz um den Ausbau existierender sowie den Aufbau zukünftiger Energierouten Richtung Mitteleuropa versetzt. ${ }^{16}$ Gleichzeitig repräsentiert der

13 Angesichts der noch Mitte der achtziger Jahre seitens der Regierung in Sofia ausgeübten massiven Repressionen gegenüber türkischstämmigen Bulgaren, die im Sommer 1989 in einem zwangsweisen Exodus von rund 300.000 Menschen muslimischen Glaubens in die Türkei gipfelten, ist die „Sensibilität“ Ankaras in dieser Frage noch heute überaus hoch.

14 Michael Lochmann: Die Türkei im Spannungsfeld zwischen Schwarzmeer-Kooperation und Europäischer Union, Zentrum für Europäische Integrationsforschung, Bonn, 2002, S. 5, abrufbar unter: http://www.zei.de/ download/zei_dp/dp_c110_lochmann.pdf.

15 Vgl. hierzu grundlegend: Directorate-General Energy and Transport, Study on Energy Supply Security and Geopolitics.

16 Wesentliche (miteinander konkurrierende) Pipelineplanungen sind dabei die Verlängerung der ukrainischen Erdölleitung Odessa-Brody in das polnische Pock, die Mitte 2005 in Betrieb gegangene und maßgeblich über türkisches Territorium führende Pipeline zwischen Baku-Tbilisi-Ceyhan (BTC) sowie die geplante Leitung zwischen den beiden türkischen Erdölhäfen Samsun und Ceyhan. Siehe hierzu ausführlich John Roberts: The Turkish Gate: Energy Transit and Security Issues, in: Turkish Policy Quarterly Nr. 4/2004. 
tankerbasierte Energietransit durch das Schwarze Meer jedoch die vorläufig spannungsgeladenste Frage zwischen den Anrainern, da aus türkischer Perspektive nicht nur ökonomische, sondern auch unmittelbar sicherheitsrelevante Interessen tangiert werden. So bildet die $325 \mathrm{~km}$ lange und an einigen Stellen lediglich 700 Meter breite Schifffahrtslinie durch den Bosporus, das Marmarameer sowie die Dardanellen für die Tankschiffe die einzige maritime Verbindung zwischen den Verladeterminals im Schwarzen Meer und den internationalen Öl- und Gashäfen. Angesichts der unmittelbaren Nachbarschaft zum rund 13 Millionen Einwohner umfassenden Großraum Istanbul nährt dies türkische Befürchtungen und Protektionswünsche. Zwar verläuft der Seeweg ausschließlich durch souveränes türkisches Hoheitsgebiet, doch findet der völkerrechtlich zulässige Regulierungsspielraum Ankaras durch die 1936 zwischen den Anrainerstaaten geschlossene „Konvention über das Meerengenstatut von Montreux"17 enge Grenzen, da diese die Einschränkung der zivilen Schifffahrt lediglich unter besonderen Umständen (etwa extreme Wetterbedingungen) erlaubt. Ungeachtet der Tatsache, dass die Frage der Umweltsicherheit sowie die damit verbundene Reduktion der ,Floating Bombs' in Anbetracht mehrerer zurückliegender Tankerunfälle ${ }^{18}$ hohe Priorität in der türkischen Politik genießt, kommt eine einseitige türkische Aufkündigung des Vertrages dennoch nicht in Betracht. Denn die Montreux-Konvention reglementiert im Gegenzug in erheblichem Maße die Passage raumfremder Kriegsmarine durch die Turkish Straits ${ }^{19}$ und garantiert der Türkei (angesichts des desolaten Zustands der ehemals dominanten sowjetischen Schwarzmeerflotte) auf lange Zeit die konventionell-militärische Überlegenheit im Schwarzen Meer. ${ }^{20}$

Stattdessen unternahm die Türkei ,in clear defiance of the Montreux Convention“21 mehrfach unilaterale Schritte zur Verschärfung der Passageregelungen, was in der jüngsten Vergangenheit wiederholt zu Spannungen zwischen den Vertragsstaaten führte. So sehen vor allem russische Beobachter in dem türkischen Vorgehen ein gegen ihre Exportinteressen gerichtetes Ansinnen. Unbestritten ist, dass die Kontrolle über die Turkish Straits ein indirektes Faustpfand für den Fall darstellt, dass es (wie zuletzt im Fall der Ukraine) mit der russischen Seite zu ernsthaften Differenzen über die Lieferung von Erdgas kommt. Hintergrund dieser strategischen Überlegung ist die extrem hohe türkische Importabhängigkeit von russischem Erdgas, die gegenwärtig mit rund $66 \%$ beziffert wird und aus türkischer Sicht langfristig auf unter $30 \%$ gesenkt werden soll. ${ }^{22}$ Angesichts dieses Umstandes ist das Interesse der Türkei an dem weiteren Ausbau ihrer Rolle als „Energy Bridge“ ${ }^{23}$ nicht nur den unmittelbar damit verbundenen ökonomischen Interessen zuzuschreiben, sondern spiegelt in erheblichem Maße auch den Wunsch Ankaras nach einer Diversifizierung der nationalen Zulieferstruktur im Energiebereich wider. Ein zentrales Element verstärkter türkischer Versorgungssicherheit bilden Planungen in Bezug auf den Iran, dessen Erdgasressourcen

17 Der Vertragstext findet sich unter: http://www.vilp.de/Depdf/d064.pdf.

18 So vor allem die folgenden Havarien: „Independenta“ (1979), „Nassia“ (1994) sowie „Volganeft-248“ (1999).

19 So regelt die Konvention in Art. 18: „Vessels of war belonging to non-Black Sea Powers shall not remain in the Black Sea more than twenty-one days, whatever may be the object of their presence there."

20 Zugleich hat es die traditionelle Landmacht Türkei in den letzten Jahren verstanden, ihre Rolle als maritimer Akteur sukzessive auszubauen und sowohl mit Hilfe eines flexiblen Stationierungskonzepts als auch der breiten Akquise moderner (zu rund 80\% aus deutscher Produktion stammender) Marinetechnologie die Grundlage ihrer heutigen Position zu legen.

21 So die offizielle Sichtweise des griechischen Außenministeriums. Vgl. http://www.mfa.gr/english /foreign_pol icy/europe_southeastern/turkey/turkeys_claims_vosporos.html.

22 Grundlegend zur türkischen Energiestruktur: International Energy Agency: Energy Policies of IEA Countries, Turkey 2001 Review, Paris 2001.

23 Energy Information Administration: Turkey, Country Analysis Brief, Department of Energy, Washington 2004, S. 1. 
über die Türkei bis in den kerneuropäischen Raum geleitet werden sollen. Aufgrund der wachsenden politischen Spannungen droht das sogenannte Nabucco-Projekt jedoch Opfer des andauernden Atomstreits mit Teheran zu werden.

\section{Der Schwarzmeerraum zwischen Konflikt und Kooperation}

\section{Türkisch-russische Beziehungstransition}

Auch jenseits der Energiefrage stellt das Verhältnis zwischen Russland und der Türkei das in vielerlei Hinsicht bedeutendste, wenngleich potenziell spannungsreichste Beziehungsgeflecht innerhalb des Schwarzmeerraums dar. Es ist seit Beginn der neugeschichtlichen Zeitrechnung nicht nur durch insgesamt 13 Kriege historisch vorbelastet, ${ }^{24}$ sondern es stellte ebenfalls während der Phase der Bipolarisierung einen Fixpunkt der global-strategischen Blockrivalität dar. Darüber hinaus versuchte die Türkei in den neunziger Jahren durch die Politisierung der Minderheitenfrage ihre Stellung als Regionalmacht auf Kosten Russlands auszuweiten, was auch in dem Vorwurf gipfelte, dass die Türkei während des ersten Tschetschenienkriegs muslimischen Rebellen einen , sicheren Hafen' gewährte und die Unterstützung von Aufständischen durch türkisch-muslimische „Wohlfahrtsorganisationen“ tolerierte. ${ }^{25}$

Andererseits entstand in Folge dieser bewegten Geschichte zwischen beiden Staaten eine besondere Form der Achtsamkeit, welche der türkischen Seite selbst angesichts der gegenwärtigen Schwäche Russlands ein erhebliches Maß an ,healthy respect“26 abverlangt. Parallel hierzu teilen die beiden größten Schwarzmeeranrainer eine Vielzahl von Gemeinsamkeiten, ${ }^{27}$ was in den letzten Jahren vor allem in sicherheitsbezogenen Fragen zu einer spürbaren Annäherung des beiderseitigen Problembewusstseins geführt hat. Als Katalysator dienten dabei in erster Linie die Ereignisse in Folge der Terroranschläge vom 11. September 2001, die nach der spannungsreichen Periode während der neunziger Jahre einen Neuanfang in den bilateralen Beziehungen ermöglichten. Seinen symbolischen Höhepunkt erfuhr das Rapprochement im Dezember 2004, als mit der Visite Putins in Ankara erstmals seit 31 Jahren wieder ein russischer Staatspräsident die Türkei besuchte. Gleichzeitig wurde bei diesem Treffen ein umfangreiches Sicherheitspaket unterzeichnet, welches nicht nur eine gemeinsame Partnerschaftsdeklaration umfasst, sondern auch den Ausbau der Kooperation im Rüstungsbereich einschließt. ${ }^{28}$ Unmittelbar auf den Schwarzmeerraum bezogen teilen die Türkei und Russland zudem das strategische Interesse, diesen vor der zunehmenden Präsenz fremder - die gegenwärtig austarierte Stabilität gefährdender - Streitkräfte abzuschotten, was sich in der Vergangenheit unter anderem darin ausdrückte, dass beide Staaten die von

24 Vgl. etwa: Zalmay Khalilzad/Ian O. Lesser/F. Stephen Larrabee: The Future of Turkish-Western Relations: Toward A Strategic Plan, Santa Monica: RAND, 2000, S. III-1, abrufbar unter: http://www.rand.org/publications/MR/MR1241/.

25 Siehe hierzu: Dmitri Trenin: Russia and anti-terrorism, in: Dov Lynch (Hrsg.): What Russia sees, Paris 2005, S. 99-114, S. 104. Auch: Aschot L. Manutscharjan: Einführung in die Grundproblematik des Tschetschenienkonfliktes, Parameter bewaffneter Konflikte, Wien 2000, S. 4, abrufbar unter: http://www.bmlv.gv.at/ pdf_pool/publikationen/manu01.pdf.

26 F. Stephen Larrabee: Turkish Foreign and Security Policy: New Dimensions and New Challenges, in: Zalmay Khalilzad/Ian O. Lesser/F. Stephen Larrabee (Hrsg.): The Future of Turkish-Western Relations, Washington 2000, S. 2.

27 So sind beide Staaten historisch als ,gescheiterte Imperien' zu betrachten, deren Landmasse sich sowohl auf dem europäischen als auch auf dem asiatischen Kontinent erstreckt und deren Sicherheit im Inneren besonders durch Separatismus, Terrorismus sowie radikalen Nationalismus herausgefordert ist. Gleichzeitig fühlen sich die Eliten beider Länder sowohl historisch als auch politisch und kulturell dem ,Europäischen Projekt' zugehörig, was in der Vergangenheit in beiden Fällen Gegenstand kerneuropäischer Zurückweisung war.

28 Report: Major Steps Forward in Turkish-Russian Relations, in: Black Sea Trend Review Nr. 9/2005, S. 30-33. 
Bulgarien und Rumänien geforderte Ausweitung der im Mittelmeer laufenden NATO-Überwachungsoperation „Active Endeavour“ auf das Schwarze Meer vehement ablehnten. ${ }^{29}$ Stattdessen zeigen sich beide Länder am Aufbau eines regionalen, ausschließlich die Schwarzmeeranrainer umfassenden Sicherheitssystems interessiert, welcher gleichzeitig die sich neu herausbildende ,Joint Hegemony' beider Staaten absichert.

Auch hinsichtlich der russischen Position in Bezug auf einen türkischen EU-Beitritt lässt sich eine Abkehr der noch vor kurzem äußerst zurückhaltenden Reaktion erkennen. Zwar ist die russische Wahrnehmung des EU-Erweiterungsprozesses weiterhin von zunehmenden Einkreisungsbefürchtungen geprägt. Deren vermutetes geostrategisches Ziel liegt in der Schaffung eines geschlossenen Grenzverlaufs zwischen der EU und Russland, was zwangsläufig auch die Inklusion der Ukraine bedeuten würde und was aus russischer Sicht unbedingt zu verhindern ist. Andererseits verbindet sich mit der Aufnahme der Türkei die stille Hoffnung von Teilen der russischen Regierung, dass der dabei zu erwartende europäisch-institutionelle Überdehnung einen irreparablen politischen Erosionsprozess der EU befördern und den Organisationsrahmen der Union auf wirtschaftliche Kernthemen reduzieren wird. ${ }^{30}$ Sollte sich diese Entwicklung bewahrheiten, könnte der türkische Beitrittsprozess gleichzeitig als Blaupause der eigenen strategischen Annäherung an die EU dienen, was Russland schon heute zu einem genauen Beobachter der türkischen Implementierung des acquis communautaire werden lässt. So ist die mehrmalige öffentliche Befürwortung des türkischen EU-Beitritts seitens des russischen Präsident Putin ${ }^{31}$ keineswegs ein Akt diplomatischer Höflichkeit, sondern trägt vielmehr auch den langfristigen russischen Interessen an einer strategischen Aufwertung der eigenen Rolle im internationalen System Rechnung, die zwangsläufig aus der Schwächung der EU resultieren würde.

\section{Russland und der post-sowjetische Einflussraum}

Der Logik Moskaus folgend, wäre ein ukrainischer EU-Beitritt bei gleichzeitiger Zurückweisung der Türkei unzweifelhaft der , Worst Case' russischer Interessenpolitik in der Region. Bezeichnete jedoch der ehemalige russische Präsident Jelzin die institutionelle Hinwendung ehemaliger Sowjetrepubliken gen Westen noch als ,casus belli“ und drohte dieser, ,[that] there is a red line for Russia which should not be crossed“32, hat sich der russische Handlungsspielraum seither dramatisch reduziert und zwingt Moskau gegenwärtig in die Rolle eines nahezu vollständig marginalisierten Zuschauers. Als vorläufig einziges Instrument, das der russischen Seite zumindest mit Blick auf die Unterbindung eines umgehenden ukrainischen NATO-Beitrittes geblieben ist, erscheint dabei das Beharren auf der, noch unter dem ehemaligen Präsidenten Kutschma vertraglich fixierten und völkerrechtlich verbindlichen bilateralen Stationierungsvereinbarung. Sie garantiert der russischen Schwarzmeerflotte die Nutzung des auf der Krim gelegenen Marinestützpunkts Sevastopol bis zum Jahr 2017. So gilt die Mitgliedschaft in der Atlantischen Allianz ohne den vorherigen vollständigen Abzug der russischen Marine von ukrainischem Territorium als nahezu ausgeschlossen. Die Regierung in Kiew drängt deshalb auf eine zeitlich vorgezogene Lösung. ${ }^{33}$ Ein Flotten-

29 Zur russischen Position vgl. Associated Press: Russia Objects to NATO Plans for Patrolling Black Sea, in: Moscow News, 10. Juni 2005.

30 Zur Gefahr der Überdehnung etwa: Erich Reiter: Die Situation der EU in ihrer geplanten strategischen Überdehnung. Sicherheitspolitische und strategische Aspekte eines Beitritts der Türkei zur EU, Büro für Sicherheitspolitik, Wien, Dezember 2004, abrufbar unter: http://www.bundesheer.at/pdf_pool/publikationen/ 06_sit_eu_tr.pdf.

31 INTERFAX: Putin hails Turkey's striving for EU membership, in: Interfax news agencies, 11. Januar 2005.

32 Reuters: Yeltsin Warns NATO Against Expansion, in: The Guardian, 15. Mai 1998. 
umzug wäre zwar in Anbetracht des gegenwärtigen Ausbaus des an der russischen Küste gelegenen Hafens Novorossiysk technisch gesehen durchaus vor 2017 möglich. Moskau verweigert jedoch kategorisch die zeitnahe Dislozierung und argumentiert ,[that] two bases are always better than one“34.

Auch in Bezug auf Georgien hat sich das Verhältnis zu Russland, welches sich trotz der prowestlich orientierten ,Rosenrevolution' im Winter 2003/04 anfänglich positiv zu entwickeln schien, spätestens seit der Krise um Süd-Ossetien (Sommer 2004) spürbar abgekühlt und geriet nach den Wahlen in Abchasien sowie der Ukraine auf einen Tiefpunkt. Im Bestreben, der als aggressiv wahrgenommenen russischen ,Hinterhof-Politik' zu begegnen und gleichfalls eine gegen Russland gerichtete Balancierungsbewegung zu initiieren, veröffentlichten die Präsidenten Georgiens und der Ukraine, Saakaschwili und Juschtschenko, deshalb im August 2005 die „Erklärung von Borjomi“, welche die Gründung einer „Community for Democratic Choice" ankündigte und sich aus den Staaten des Ostsee-, des Schwarzmeerraums und des Kaspischen Meeres rekrutieren soll. Das erklärte Ziel ist die Eröffnung einer „,new era of democracy, security, stability and lasting peace“ in der Region. Allerdings fehlt der Initiative, ebenso wie der unter Beteiligung beider Staaten entstandenen GUUAM-Staatengruppe, jedwedes politisches Profil, was die Bestrebungen ebenso ehrgeizig wie aussichtslos erscheinen lässt. Nichtsdestotrotz muss die russische Seite spätestens seit den revolutionären Wandlungen in beiden Staaten und ungeachtet der friktionsbehafteten Ausläufer eines postimperialen Zerfallsprozesses konstatieren, dass sich ,eine wachsende Konkurrenz mit der EU um Einfluss und Integration [in diesem Raum vollzieht] und eine fortschreitende Erosion seiner schon zuvor angefochtenen Dominanz innerhalb des GUS-Raums“"35 zu Tage tritt.

\section{Bulgarien, Rumänien und der wachsende Einfluss der USA}

Als eine weitere Front im Ringen um die regionale Vorherrschaft entpuppen sich zunehmend auch die unterschiedlichen Vorstellungen der Anrainerstaaten in Bezug auf die zukünftige Sicherheitsarchitektur im Schwarzmeerraum. So bemüht sich vor allem Rumänien seit der Amtsübernahme von Präsident Traian Basescu im Dezember 2004 verstärkt um den Einflusszuwachs seines Landes in der Region, die nach der EU zur zweiten Priorität der rumänischen Außenpolitik erhoben wurde. ${ }^{36}$ Bereits die Vorgängerregierung hat durch den Kauf von zwei ausgemusterten britischen Fregatten im Jahr 2002 den Grundstein dafür gelegt, mittels militärischer Präsenz zukünftig zu einer dominierenden Kraft heranzuwachsen. Parallel sucht die Regierung in Bukarest gemeinsam mit der bulgarischen Seite das Schwarze Meer sukzessiv zu internationalisieren und (beflügelt durch die Entwicklungen in der Ukraine und Georgien) mit Hilfe der EU und der NATO eine aktive Stabilitäts- und Sicherheitsexportpolitik zu initiieren. Nachdem die beiden Balkanstaaten jedoch selbst innerhalb der NATO mit der Forderung nach einer Ausweitung der Operation ,Active Endeavour' auf das Schwarze Meer wenig Gehör fanden, setzte die Regierung Basescu zwischenzeitlich sogar auf den Aufbau einer autonomen „Achse Bukarest-London-Washington“ ${ }^{“ 37}$, die allerdings nicht nur die

33 Vgl. hierzu: Ulrich Schmid: Neue Reibungen um die Schwarzmeerflotte. Kiews Nato-Ambitionen sorgen für Spannung mit Moskau, in: Neue Zürcher Zeitung, 27. April 2005.

34 So der russische Verteidigungsminister Sergei Ivanov. Zitiert in: Jeremy Page: Ukraine could sink Russia fleet, in: The Times, 6. Dezember 2004.

35 Halbach, Der Kaukasus in neuem Licht, S. 18.

36 Vgl. Günter W. Dill/Maria Vasiu: Im Fadenkreuz rumänischer Außen- und Sicherheitspolitik: die Schwarzmeerregion, Konrad-Adenauer-Stiftung, Bukarest, November 2005, abrufbar unter: http://www.kas.de/ db_files/dokumente/7_dokument_dok_pdf_7719_1.pdf. 
strategische Orientierung des Landes, sondern auch die maßlose Selbstüberschätzung der rumänischen Regierung unterstrich. Wurde von diesen Plänen bereits wieder Abstand genommen, bleibt die Stationierung US-amerikanischer Truppen auf rumänischem Boden (Kogalniceanu, Babadag) ${ }^{38}$ weiterhin ein wesentliches Element der eigenen Rollenaufwertung und stößt zwangsläufig auf vehementen russisch-türkischen Widerspruch. ${ }^{39}$ Mag diese Strategie aus Sicht eines Landes wie Rumänien durchaus verständlich erscheinen, sind die geopolitische „Raumfremde“ der Vereinigten Staaten sowie das stets zwischen „Patron“ und „Günstling“" nachweisbare Axiom ,pro protectione oboedientia“40 nichtsdestotrotz für die Region als strukturelle Konflikttriebfedern zu betrachten. Bereits heute werden ,reports of TurkishRussian discussions of a coordinated policy in the Black Sea region" seitens führender amerikanischer Analysten als ,,most worrying“ eingestuft und vor allem der Türkei ein ,,negative regional behavior" unterstellt. ${ }^{41}$ Aus Sicht der EU steht dabei besonders zu befürchten, dass die USA nach der neuerlichen Osterweiterung versucht sein könnten, über beide Balkanstaaten aktiv auf die Willensbildung der Union in der Frage der zukünftigen Schwarzmeerpolitik Einfluss zu nehmen und mit deren Hilfe eine allzu selbstbewusste Außen- und Sicherheitspolitik der Union bereits innerhalb europäischer Gremien auszubremsen. ${ }^{42}$

\section{Regionale Kooperationsansätze}

Ungeachtet der latenten Konfliktlagen und Interessendivergenzen, kam es bereits kurz nach dem Aufbrechen der bipolaren Weltordnung sowie dem damit einhergehenden Wegfall der während der Phase des Kalten Krieges zwischen den Anrainerstaaten vorherrschenden strukturellen Kooperationsbarriere zu einem sukzessiven Aufbau gemeinsamer Organisationsstrukturen. Die Initiative für die schwarzmeerbezogene Kooperation ging dabei maßgeblich von der Türkei aus, die nach der offensiven Zurückweisung des türkischen Gesuchs um die Aufnahme von Beitrittsgesprächen seitens der EG-Kommission im Dezember 1989 nach neuen außenpolitischen Aktionsfeldern suchte. ${ }^{43}$ Gleichzeitig sah die vom damaligen türkischen Außenminister Ismail Cems formulierte „,neue ,Grand Strategy’ der Türkei [...] vor, dass diese nicht mehr an der Peripherie Europas, sondern im Zentrum der euro-asiatischen Region liegt“"44, was sich neben den Versuchen der Reaktivierung des Panturkismus gleichfalls in ihrem schwarzmeerbezogenen Ansatz ausdrückte. Im Mittelpunkt der regionalen Institutionen steht die ,Black Sea Economic Cooperation Organization' (BSEC), welche 1992 von Albanien, Armenien Aserbaidschan, Bulgarien, Georgien, Griechenland, Moldau, Ru-

37 Basescu zitiert in: Achse Bukarest-London-Washington „keine Rhetorik“, in: Allgemeine Deutsche Zeitung für Rumänien, 19. Januar 2005.

38 Vgl. US-Romania nearing Black Sea base deal, in: The New Anatolian, 27./28. August 2005.

39 Grundlage der US-amerikanischen Stationierungsplanungen ist dabei der aktuelle „US Global Defense Posture“, der eine effektivere Machtprojektion in der erweiterten Region erlauben soll. Vgl. hierzu: Department of Defense: Strengthening US Global Defense Posture, Report to Congress, Washington, D.C., 17. September 2004, abrufbar unter: http://www.defensecommunities.org/ResourceCenter/Global_Posture.pdf.

40 So sah bereits Thomas Hobbes das Schutz-und-Gehorsam-Axiom als so elementar an, dass er sich in seinem opus magnum ausdrücklich und ausschließlich der Aufdeckung dieses Beziehungsgeflechts widmete. Vgl. Thomas Hobbes: Leviathan, Neuwied 1966, S. 544.

41 Beide Zitate: Bruce Pitcairn Jackson: The Future of Democracy in the Black Sea Region, Testimony Before the Committee on Foreign Relations Subcommittee on European Affairs, 8. März 2005, S. 4, abrufbar unter: http://www.senate.gov/ foreign/testimony/2005/JacksonTestimony050308.pdf.

42 Für eine ebenfalls prominente, explizit US-amerikanische Sichtweise auf die Region siehe: Ronald D. Asmus: Developing a New Euro-Atlantic Strategy for the Black Sea Region, in: Insight Turkey Nr. 3/2006, S. 43-65.

43 Hierzu etwa: Lochmann, Die Türkei im Spannungsfeld, S. 3-4.

44 Hüseyin Bagci: Die Problematik der türkischen „Grand Strategy“ in einer sich verändernden Sicherheitsumwelt: Gestern und Heute, in: Landesverteidigungsakademie Wien (Hrsg.): Studien und Berichte zur Sicherheitspolitik 2000, Wien 2000, S. 5-24, S. 10. 
mänien, der Russischen Föderation, der Türkei sowie der Ukraine ${ }^{45}$ ins Leben gerufen wurde ${ }^{46}$. Auf ihrem gemeinsamen Gipfel in Istanbul verabschiedeten die Staats- und Regierungschefs hierzu eine „Deklaration zur Schwarzmeerkooperation“ deren Ziel darin bestand „to ensure that the Black Sea becomes a sea of peace, stability and prosperity, striving to promote friendly and good-neighborly relations “47. Erwogene Handlungsoptionen waren unter anderem Sicherheits- und Vertrauensbildende Maßnahmen, Dialog und Kooperation oder auch die handelspolitische Verflechtung. Parallel dazu wurde mit dem „Bosphorus Statement" ein Dokument verabschiedet, in welchem neben ökonomischen auch ausdrücklich sicherheitspolitische Fragen der Region aufgegriffen und vor der wachsenden Gefahr regionaler Krisen gewarnt ${ }^{48}$ wurde. Auf dem 1998 in Jalta stattfindenden Gipfel verabschiedeten die Staatenvertreter zudem eine gemeinsame Charta, durch welche die BSEC offiziell in den Rang einer Internationalen Organisation erhoben wurde und seither nicht nur über ein ,Permanent International Secretariat' (PERMIS) in Istanbul, sondern ebenfalls über die Schwarzmeerbank für Handel und Entwicklung in Thessaloniki verfügt ${ }^{49}$. Jenseits dessen konnten sich die Mitgliedsstaaten bisher jedoch nur über wenige operative Maßnahmen verständigen, was den politischen Nutzen von BSEC selbst in den Augen von hochrangigen PERMIS-Mitarbeitern hat schwinden lassen. Erschwert wird die BSEC-interne Konsensfindung in den letzten Monaten zudem durch die russische Seite, welche in Folge ihres allmählich schwindenden regionalen Einflusses sowie der zunehmend US-amerikanische Präsenz in eine klassische Blockadehaltung zu verfallen droht. So blockierte Russland unter anderem einen, von den USA gestellten BSEC-Aufnahmeantrag mit seinem Veto, wohingegen das seitens der EU ins Auge gefasste Bestreben, innerhalb des Schwarzmeerkooperationsrats mittelfristig den Status eines ,permanenten Beobachters' zu erhalten, weiterhin als aussichtsreich $^{50}$ gilt.

Durchaus konstruktiv gestaltet sich die russische Politik hingegen in der ebenfalls auf die Initiative Ankaras zurückgehenden ,Black Sea Naval Co-operation Task Force' (BLACKSEAFOR), der darüber hinaus auch die Staaten Bulgarien, Georgien, Rumänien sowie die Ukraine angehören. Erklärtes Ziel der im April 2001 gegründeten ,On-Call Force' ist es ,to contribute to the further strengthening of friendship, good relations and mutual confidence among the Black Sea littoral states as well as to improve peace and stability in the region, through the enhancement of co-operation and interoperability among the naval forces." 51 Hierzu führen die Teilnehmerstaaten halbjährlich gemeinsame, paritätisch zusammengesetzte Manöver durch, in welchen etwa die Zusammenarbeit der Seestreitkräfte im Bereich der Seerettung oder auch in der Minensuche geübt wird. Sind dabei vor allem Russland und die Türkei an der Errichtung fester Strukturen sowie der Etablierung eines stehenden Haupt-

45 Serbien und Montenegro traten der Organisation 2004 bei.

46 Für eine Übersicht weiterer Gremien und Initiativen siehe: Robert F. Simmons: Regional Security and Defence Co-Operation in the Black Sea Area, Political-Military Steering Committee on Partnership for Peace, North Atlantic Council, Brussels, 31. Januar 2005.

47 So der Wortlaut von Punkt 8 der Summit-Declaration on Black Sea Economic Cooperation.

48 Hierzu: Simmons, Regional Security and Defence Co-Operation in the Black Sea Area.

49 Zur Entwicklungsgeschichte siehe: Nurver Nures: Die Schwarzmeerwirtschaftskooperation (SMWK) am Scheideweg, in: KAS-Auslandsinformationen, Konrad-Adenauer-Stiftung Nr. 8/2002, S. 46-69.

50 So kam es am 11. April 2005 zwischen der EU und BSEC zu einem ersten Gedankenaustausch darüber, wie die „EU could participate on a more regular basis in BSEC“. Vgl. "Black Sea Area and Euro-Atlantic Security: Strategic Opportunities", Contribution by Dr. Norbert Baas, EU Special Envoy of the German Foreign Office for Eastern Europe, Central Asia and the Southern Caucasus, Conference Proceedings, Bucharest, 20. April 2005.

51 So der Wortlaut des Art. IV, 1 des Agreement on the Establishment of the Black Sea Naval Co-operation Task Group. 
quartiers zum Zwecke der kontinuierlichen Übernahme von maritimen Sicherheitsaufgaben interessiert, wird dies besonders von Bulgarien und Rumänien abgelehnt, da beide weiterhin die Präsenz der NATO in der Region favorisieren.

\section{Die strategische Interessenlage der Europäischen Union}

Auf Grundlage der vorangegangenen Betrachtung lassen sich mehrere strategische Kernthemen destillieren, die innerhalb eines erweiterten Sicherheits- und Interessenspektrums Relevanz für die EU besitzen. ${ }^{52}$ Gleichzeitig wird die langfristige Wertschöpfung in diesen Bereichen maßgeblich durch das zukünftige europäische Verhältnis zur Türkei determiniert, ${ }^{53}$ was die EU im Beitrittsprozess auch zu Zugeständnissen und Rücksichtnahmen gegenüber dem Kandidatenland zwingt. Angesichts einer rapide wachsenden Importabhängigkeit der EU im Erdöl- (2002: 76\%, 2030: 94\%) und Erdgas- (2002: 49\%, 2030: 81\%) Bereich, welche das Potenzial besitzt, die politische Handlungsautonomie der EU-Staaten erheblich zu gefährden, stehen Überlegungen zum Energietransit sowie der Schaffung diversifizierter Zulieferstrukturen im Mittelpunkt der mit dem Schwarzmeerraum verknüpften europäischen Interessen ${ }^{54}$. Dies umfasst im Besonderen die weitere Erschließung nichtrussischer Energieressourcen im kaspischen Raum (Turkmenistan, Kasachstan) als auch den Abschluss langfristiger Lieferverträge mit dem Iran. Der Türkei kommt die Rolle eines ,Major Energy Hub` zu, wenngleich das Beispiel der Mitte 2005 eröffneten BTC-Pipeline verdeutlicht, dass Ankara in der Energietransit-Frage weit mehr ,Free Rider' als handlungsleitender Akteur ist. Sie profitiert in erster Linie von ihrer zentralen geographischen Lage, den auf Diversifizierung ausgelegten Energiestrategien europäischer und nordamerikanischer Staaten sowie den langfristigen und ausschließlich von ökonomischen Interessen geleiteten Investitionsüberlegungen der (ehemals) „Seven Sisters ${ }^{55 “ “}$

Eng mit der Ressourcenfrage ist aus Sicht der EU auch der Sicherungsgedanke eines „geopolitischen Vorhofs“ in der Region Zentralasien verbunden. War das unmittelbar an das Schwarze Meer angrenzende Gebiet bisher ein Raum minderer geostrategischer Bedeutung, bildet dieser aufgrund der engen Verwobenheit mit dem Kaukasus und den zentralasiatischen Republiken mittlerweile einen strategisch umkämpften Korridor zur ,Schatzkammer Asiens', die auch den Mittleren Osten einschließt. ${ }^{56}$ Das gewachsene Interesse der EU steht auch in Zusammenhang mit dem in der EU-Sicherheitsstrategie explizit ausformulierten Selbstanspruch eines ,globalen Akteurs“"57, dessen Rolle sich erst dann adäquat ausfüllen lässt, wenn die EU auch in dieser bedeutenden Weltregion zu einer aktiven Interessenartikulation fähig ist. ${ }^{58}$ Wie in der Energiefrage ist die Union auch hier auf die langfristige Einbin-

52 Grundsätzlich hierzu etwa: Heinz Kramer: EU-kompatibel oder nicht? Zur Debatte um die Mitgliedschaft der Türkei in der Europäischen Union, SWP-Studie Nr. 34, August 2003, Berlin, S. $27-32$.

53 So bemerkt etwa Kramer, dass „sich im Falle einer Ablehnung auch jene sicherheitspolitisch-strategischen Vorteile schwerer verwirklichen [lassen], die Befürworter eines Beitritts häufig anführen". Ebenda, S. 30.

54 Vgl. International Energy Agency: World Energy Outlook 2004, Paris: IEA, 2004. Für eine grundlegende Betrachtung zum Komplex der EU-Importabhängigkeit siehe: Directorate-General Energy and Transport: Study on Energy Supply Security and Geopolitics.

55 Hierunter versteht man die ehemals sieben führenden Mineralölkonzerne, von denen nach mehreren Fusionen heute lediglich ExxonMobil, ChevronTexaco, Shell und BP existieren.

56 So unterstreicht etwa Jackson die Bedeutung der Region wenn er schreibt: „Every nineteenth century European power understood that the nation which controlled the Black Sea could control the most important real estate in the Middle East." Bruce Pitcairn Jackson, The Future of Democracy in the Black Sea Region, S. 2.

57 Europäischer Rat: Ein sicheres Europa in einer besseren Welt. Europäische Sicherheitsstrategie, Brüssel, 12. Dezember 2003, S. 1.

58 Bisher findet die Region jedoch selbst in der EU-Sicherheitsstrategie keinerlei Erwähnung. Auch existieren bisher keine GASP-Stellungnahmen, die unmittelbar auf den Schwarzmeerraum Bezug nehmen. 
dung der Türkei angewiesen, da diese in Hinblick auf Zentralasien ,den politischen Einfluss der EU in dieser Region kanalisieren helfen [könnte] "59 und über die südliche (über Rumänien, Bulgarien und die Türkei führende) Landtrasse einen ungehinderten physischer Zugang gesichert würde. Als (im konventionellen Bereich) militärisch stärksten Schwarzmeeranrainer würde die Türkei zugleich einen strategischen Vorposten der EU bilden, der zudem über ein äußerst solides diplomatisches Netzwerk in die Region verfügt.

Parallel hierzu bilden die im ,Erweiterten Schwarzmeerraum' weiterhin vorherrschenden Regionalkonflikte einen willkommenen Testfall für die Europäische Sicherheits- und Verteidigungspolitik (ESVP), da sich spätestens mit dem Näherrücken des türkischen Beitritts die bisher äußerst zurückhaltende Politik der EU gegenüber den mit Russland verbundenen ,Frozen Conflicts' nicht länger aufrecht erhalten lassen wird. Vielmehr erzeugt die erweiterungsbedingte geographische Annäherung der EU an diese Territorien einen steigenden Konfliktlösungsdruck, der eine weitere entscheidende Wegmarke der Union hin zu einem tatsächlich globalen Akteur im Krisenmanagementbereich bedeuten könnte. Perspektivisch vermag die EU auch auf das diplomatische Netzwerk der Türkei zurückgreifen und hierüber beispielsweise moderierend auf die Staaten Georgien und Aserbaidschan Einfluss zu nehmen. Langfristig anzustreben ist ein umfassender europäischer Systemexport in den Schwarzmeerraum, welcher zur regionalen Stärkung von Demokratie, Rechtstaatlichkeit und Menschenrechten beiträgt und den Zielen der Gemeinsamen Außen- und Sicherheitspolitik der Union (Art. 11 EU-Vertrag) entspricht.

Ein gänzlich anderes Interessenfeld eröffnet sich bei der Frage nach der freie Passage durch die Turkish Straits, die trotz vertraglicher Garantien im Rahmen der Montreux-Konvention einer zunehmenden ,Selbstregulierung' durch die Türkei ausgesetzt sind und unmittelbar die strategischen Interessen aller großen westlichen Staaten einschließlich der mit ihnen assoziierten Bündnissysteme berühren. Die Sicherung des Prinzips ,Freiheit der Meere' steht dabei nicht nur für das Verlangen nach einem ungehinderten tankerbasierten Energietransit, sondern zugleich für die Aufrechterhaltung der militärischen Überlegenheit des Westens, dessen maritime Mobilität seit jeher das strategische Rückgrad weltweiter Machtprojektion bildet. Um einen von der Türkei ausgehenden Dominoeffekt in der Frage der unilateralen Regulierung strategischer Wasserstraßen (Panamakanal, Straße von Hormuz, Suezkanal, Straße von Malakka) zu verhindern, muss die EU diesen Streitpunkt zwischen den Schwarzmeeranrainern aktiv moderieren und einen fairen Interessenausgleich befördern.

Vornehmlich mit dem Ziel der Stärkung der inneren Sicherheit der Union ist hingegen der Auf- und Ausbaus eines effektiven EU-Grenzregimes in der Region verknüpft, da die sowohl durch Bulgarien als auch in Teilen durch Rumänien führende ,Balkanroute' nicht nur die Hauptachse des aus Zentralasien über die Türkei in den Schengenraum geleiteten Drogenverkehrs darstellt, sondern ebenfalls ein bedeutender Transitweg illegaler Migranten repräsentiert. ${ }^{60}$ Stärker als zuvor wird die Union somit nach der bevorstehenden zweiten EU-Osterweiterung auf die Zusammenarbeit mit der Türkei aber auch anderen Schwarzmeeranrainern bei der Grenzsicherung angewiesen sein.

59 Europäische Kommission: Arbeitsdokument der Kommissionsdienststellen, Fragen im Zusammenhang mit der möglichen Mitgliedschaft der Türkei in der Europäischen Union, COM(2004)656 final, S. 7.

60 Vgl. hierzu: Ebenda, S. 45-50. 


\section{Szenarien jenseits der türkischen Beitrittsfinalisierung}

Angesichts des nur skizzierten strategischen Interessenprofils der EU wird sich eine umfassende Eröffnung europäischer Handlungsoptionen im Schwarzmeerraum am besten auf dem Weg einer möglichst breiten Einbindung der türkischen Seite realisieren lassen. Zwar existiert historisch kein Beispiel dafür, ein einmal eröffnetes Beitrittsverfahren seitens der EU nicht abzuschließen, doch bilden die europäisch-türkischen Gespräche zweifellos ,,Verhandlungen ohne Vorbild“61. So hält bereits der Verhandlungsrahmen fest: „These negotiations are an open-ended process, the outcome of which cannot be guaranteed beforehand." 62 Die Gefahr des Scheiterns geht von beiden Seiten aus, da auch von türkischen Führungskräften betont wird, dass die Türkei „das Recht hat, ,Ja' oder ,Nein’ zu sagen““63

Kommt es bei der Prognose strategischer türkischer Reaktionsmuster im Falle einer Zurückweisung in erheblichem Maße auf die jeweiligen Umstände der Ablehnung an (die Spannweite reicht vom frühzeitigen Abbruch der Verhandlungen bis hin zum Scheitern in ,letzter Sekunde' durch eine Volksabstimmung), muss angesichts des langfristigen Charakters einer europäischen Zurückweisung im Gegensatz zur erstmaligen Ablehnungsbescheid vom Dezember 1989 von einem erheblichen anti-europäischen ,Blowback' ausgegangen werden. Als wahrscheinlich gilt dabei nicht nur die kurzfristige Radikalisierung der türkischen Innenpolitik sowie die Stärkung nationalistischer (möglicherweise auch religiös motivierter) Kräfte, sondern auch eine langfristige, gegen die EU-gerichtete Balancierungsbewegung. Wäre eine strategische türkisch-amerikanische Wiederannäherung als die wahrscheinlichste Option zu erachten, kann im Falle des Scheiterns auch ein türkisch-russisches Zweckbündnis nicht ausgeschlossen werden. Wie zuvor in den neunziger Jahren wird die Türkei zudem den Versuch unternehmen, eine strategische Allianz mit den Turkstaaten Aserbaidschan, Kasachstan, Usbekistan, Turkmenistan und Kirgistan zu schmieden, wobei Ankara im Gegensatz zu vorangegangenen Initiativen bereits heute auf erhebliche diplomatisch-politische Vorarbeiten aufbauen kann. Wäre diese Entwicklung aus Sicht der EU mit erheblichen Abstrichen hinsichtlich der Durchsetzung der oben dargelegten strategischen Interessen verbunden, bedeutet dies jedoch nicht zwangsläufig eine sicherheitspolitische Destabilisierung des Schwarzmeerraums.

Ein weiteres Alternativszenario zur Vollmitgliedschaft gliedert sich in zwei unterschiedliche Gedankenmodelle und skizziert die Situation „If Turkey Says No“64. Das erste Konstrukt nimmt dabei die Annahme zur Grundlage, dass die Türkei in Folge einer durchaus rationalen und interessenbezogenen Analyse ihren bisherigen Standpunkt revidiert, wonach lediglich eine Vollmitgliedschaft den Endstatus zur EU definiert. Stattdessen könnte Ankara auf die Übernahme einer unabhängigeren, sowohl europäisch als auch asiatisch ausgerichteten Akteursrolle spekulieren und nach Abschluss zahlreicher bilateraler Übereinkünfte mit der EU lediglich eine konsolidierte ,strategische Partnerschaft' eingehen. Zugleich würde die Türkei ihre Rolle als ,euro-asiatischer Gatekeeper' in vollem Maße zu ihren Gunsten ausnutzen und könnte (im Gegensatz zum Zurückweisungs-Szenario) beim weiteren Ausbau des Beziehungsgeflechts zu den Turkstaaten sowie langfristig zum Iran aus einer Position

61 Heinz Kramer: EU-Türkei: Vor schwierigen Beitrittsverhandlungen, Stiftung Wissenschaft und Politik, Berlin, Mai 2005, S. 7.

62 Negotiating Framework for Turkey. Principles governing the negotiations, 3. Oktober 2005, S. 1, abrufbar unter: http://www.eu.int/comm/enlargement/docs/pdf/st20002_en05_TR_framedoc.pdf.

63 Hilimi Özkök: Jährliche Bewertungsrede des Generalstabschefs an der Kriegsakademie, Ankara, 20. April 2005, S. 21

64 Sedat Laciner: If Turkey Says No?, in: Turkish Weekly (17. Juli 2005), abrufbar unter: http://www.turkishweekly.net/editorial.php?id=16. 
der Stärke heraus agieren. Sowohl für den Schwarzmeerraum und seine Anrainer als auch für die Staaten der EU würden sich hingegen auf strategischer Ebene im Vergleich zur heutigen Situation lediglich geringfügige Veränderungen ergeben. Aus Sicht Moskaus bliebe zudem die befürchtete südliche Einkreisungsbewegung aus und würde auch georgische Hoffnungen auf einen etwaigen EU-Beitritt negieren, da diese nur bei direkter territorialer Anbindung realistisch erscheint.

Grundlegend unterschiedlich gestalten sich hingegen die Perspektiven im Falle einer nationalistischen Reflexen folgenden Abwendung der Türkei vom Ziel der Vollmitgliedschaft. Müsste man wie im Falle einer europäischen Zurückweisung mit erheblichen Abstrichen bei der Durchsetzungsfähigkeit der eigenen Interessen in der erweiterten Region rechnen, ergibt sich auch aus Sicht der übrigen Schwarzmeeranrainer eine keineswegs wünschenswerte Konfrontation, da vor allem die russische Seite empfindlich auf jedwede Form nationalistischer oder religiös-motivierter Tendenzen reagieren dürfte und somit die Gefahr regionaler Destabilisierung birgt. Gleichzeitig wäre eine strategische ,Absicherung' zwischen der EU und Russland eine keineswegs fern liegende Konsequenz. Ist das Risiko eines solchen Entwicklungsverlaufs zwar gegenwärtig nicht als akut zu charakterisieren, lässt sich - angesichts des ,unwürdigen Feilschens" 65 im Vorfeld der offiziellen Aufnahme der EU-Beitrittsgespräche - bereits heute feststellen, dass sowohl in der Bevölkerung als ,auch bei der türkischen Regierung die Beitrittseuphorie verflogen ist."66

\section{Ausblick}

Im Zuge der laufenden Beitrittsprozesse von Bulgarien, Rumänien und der Türkei sowie der sich kontinuierlich zuspitzenden Frage nach der langfristigen europäischen Versorgungssicherheit im Energiebereich ist auch der bisweilen als ,europäischer Blinddarm ' verspottete Schwarzmeerraum zunehmend in den Interessenfokus der EU gerückt. Bislang existiert auf Seiten der EU jedoch kein übergreifender raumbezogener Ansatz. Stattdessen konzentrierte sich die Union vorwiegend auf den Aufbau bilateraler Beziehungsmuster im Rahmen ihrer unterschiedlichen Kooperationsinstrumente und Beitrittsfahrpläne und beschränkte sich bei der Behandlung regionaler Fragen auf wenige vornehmlich technische Übereinkünfte. ${ }^{67}$ So findet der Schwarzmeerraum als Problem- und Interessengebiet vorläufig weder in der EU-Sicherheitsstrategie noch in einzelnen GASP-Stellungnahmen Erwähnung. Künftig wird die effektive Interessenwahrnehmung in dieser Region jedoch stark davon abhängen, ob es der EU gelingen wird, eine gemeinsame strategische Linie für den gesamten euro-asiatischen Grenzraum zu entwickeln. Dies gilt vor allem für den Bereich sicherheitsrelevanter Fragen, da die Vielzahl fortexistierender Spannungen und Konflikte den Aufbau eines autochthonen funktionsfähigen multilateralen Institutionengefüges in dieser Region auf absehbare Zeit behindern. Auf der anderen Seite bleibt festzustellen, dass vor allem die zunehmende politisch-ökonomische Interdependenz der Anrainerstaaten einen ,neutralisierenden Kitt“ darstellt, welcher sowohl aktuelle als auch künftige Divergenzen in positiver Weise moderieren wird und gleichfalls das Risiko eines (mit dem türkischen Beitritt zu befürchteten), Entrapment" der EU in regionale Konfliktlagen eindämmen hilft. Besonders hervorzuheben ist zudem das sich seit mehreren Jahren stetig verbessernde Verhältnis zwi-

65 Raúl Lautenschütz: Unwürdiges Feilschen um das Türkei-Mandat, in: Neue Zürcher Zeitung, 3. Oktober 2005.

66 Heinz Kramer: Türkei-Verhandlungen als Spielball der Interessen, in: SWP-Aktuell Nr. 42, September 2005, S. 1, abrufbar unter: http://www.swp-berlin.org/common/get_document.php?id=1392.

67 Hierzu zählen etwa die Programme: Interstate Oil and Gas Transport to Europe (INOGATE), Transport Corridor Europe-Caucasus-Asia (TRACECA) sowie das Black Sea Pan-European Transport Area (PETrA). Vgl. Vahl, The EU and Black Sea Regional Cooperation. 
schen den einstigen Erbfeinden Türkei und Russland, das sich maßgeblich auf ein Gerüst neuer gemeinsamer Interessen in der Region abzustützen weiß. Gleichzeitig wird jedoch die ,Joint Hegemony' beider Staaten von den restlichen Anrainern, die weder über entsprechende militärische Fähigkeiten noch über einen sich hieraus ableitenden strategischen Einfluss im Schwarzmeerraum verfügen, mit erheblicher Skepsis betrachtet. Der Wunsch nach größerer regionaler Mitsprache wird in erster Linie am Beispiel des künftigen EU-Mitglieds Rumänien deutlich, das nicht nur aus eigener Kraft an militärischem Profil zu gewinnen sucht, sondern sich vor allem durch eine affirmative Politik in Bezug auf US-amerikanische Stationierungsinteressen in der Region einen Macht- und Sicherheitsgewinn verspricht. Ist das Aufgehen der rumänischen Reziprozitätskalkulation dabei durchaus fraglich, herrscht im Gegenzug jedoch wenig Zweifel daran, dass dieses Handeln mittelfristig neuen Konfliktstoff in die Region befördern könnte. Anzeichen hierfür finden sich bereits heute und schlagen sich in erster Linie im türkisch-amerikanischen Verhältnis nieder. So lässt es durchaus aufhorchen, wenn selbst führende Diplomaten die bilateralen Beziehungen als zunehmend ,allergic" 68 charakterisieren und einflussreiche US-Regierungsberater über den einst so engen europäischen NATO-Partner bemerkt: „Turkey has entered a dangerous period both for itself and for US-Turkish relations which deserves serious attention." 69

In Bezug auf die Ukraine ist angesichts der äußerst volatilen politischen Situation vorerst keine abschließende Verortung dieses Landes in der regionalen Gesamtkonstellation möglich. Gleichwohl weisen auch hier einzelne Entwicklungsaspekte auf einen zunehmenden Verdruss Kiews gegenüber der wachsenden russisch-türkischen Dominanz im Schwarzmeerraum hin. So wird der EU zukünftig eine besondere Sensibilität in der Frage des regionalen Interessenausgleichs abverlangt werden und eine aktive Einbringung politischer und diplomatischer Initiativen erforderlich machen. Eine Möglichkeit hierzu bietet sich unter anderem durch die angestrebte Partizipation der EU am Schwarzmeerkooperationsrat, in welchem die Union ihren reichhaltigen Erfahrungsschatz bei Fragen des Institutionenaufbaus sowie der multilateralen Konsensfindung einbringen kann.

Um dieser Rolle als Antriebsfeder gerecht zu werden, kommt die EU jedoch nicht umhin, gegenüber den Anrainerstaaten mittels einer transparenten regionalen Agenda an Glaubwürdigkeit als ehrlicher Makler zu gewinnen und die bisher stark bilateral orientierten Beziehungsmuster zu erweitern. Ein erster Schritt hin zu einer kohärenteren europäischen Linie wäre dabei die Entwicklung einer gemeinsamen EU-Regionalstrategie für den Schwarzmeerraum, die nach Vorbild bereits existierender Grundlagendokumente ${ }^{70}$ den Interessenund Handlungsfokus der Union von der einzelstaatlichen Ebene löst sowie unterschiedliche Politikbereiche (Energie, Sicherheit, Grenzkontrolle et cetera) zu bündeln sucht.

68 So der ehemalige US-Botschafter in der Türkei Mark Parris: Allergic Partners: Can US-Turkish Relations Be Saved?, in: Turkish Policy Quarterly, Nr. 1/2005, S. 49-58.

69 Bruce Pitcairn Jackson, The Future of Democracy in the Black Sea Region, S. 4.

70 So etwa die Gemeinsame Strategie für die Mittelmeerregion (2000/458/GASP). Eine Gemeinsame Strategie für die Region Westlicher Balkan befindet sich gegenwärtig in Entwicklung. 